\title{
Effects of GGCX overexpression on anterior cruciate ligament transection-induced osteoarthritis in rabbits
}

\author{
XIAOLING FU ${ }^{1}$, RUIYUN QIU ${ }^{2}$, CHUNFANG TANG $^{3}$, XIAOMEI WANG ${ }^{1}$, XIGAO CHENG $^{1}$ and MING YIN ${ }^{1}$ \\ ${ }^{1}$ Department of Orthopedics, The Second Affiliated Hospital of Nanchang University, Nanchang, Jiangxi 330006; \\ ${ }^{2}$ College of Information Engineering, Nanchang University, Nanchang, Jiangxi 330031; ${ }^{3}$ Department of Orthopedics, \\ The First Affiliated Hospital of Gannan Medical University, Nanchang, Jiangxi 341099, P.R. China
}

Received September 28, 2016; Accepted October 11, 2017

DOI: $10.3892 / \mathrm{mmr} .2017 .8304$

\begin{abstract}
Effective therapeutic methods for osteoarthritis (OA) are lacking. $\gamma$-glutamyl carboxylase (GGCX) is a key enzyme that regulates carboxylation of cartilage matrix Gla protein (MGP). Whether GGCX overexpression protects against OA remains unknown. The aim of the present study was to explore the effects of GGCX overexpression on anterior cruciate ligament transection (ACLT)-induced OA and its mechanisms in Japanese white rabbits. ACLT surgery was used to establish an OA model in rabbits. A total of 48 rabbits were randomly divided into 4 groups: Sham, OA model + GGCX overexpression plasmid, OA model + saline and OA model + empty vector. The expression of uncarboxylated MGP (ucMGP), carboxylated MGP (cMGP), matrix metalloproteinase (MMP)-13, collagen type $X$, collagen type II, tumor necrosis factor (TNF)- $\alpha$ and interleukin (IL)-1 $\beta$ were detected by ELISA, immunohistochemistry, reverse transcription-quantitative polymerase chain reaction and western blotting. Morphological changes to tibial cartilage were assessed by Giemsa and safranin O-fast green staining, respectively. Compared with the Sham control, GGCX expression was significantly decreased in the OA Model group. GGCX expression was increased by injection of a lentivirus-carried overexpression plasmid that encoded GGCX. GGCX overexpression ameliorated ATLC-induced damage in articular cartilage. OA Model rabbits exhibited significantly decreased expression levels of cMGP and collagen type II, and increased expression of ucMGP, collagen type X, MMP-13, IL-1 $\beta$ and TNF- $\alpha$. Notably, these expression levels were reversed by GGCX overexpression in OA Model rabbits. Results from the present study indicated that GGCX expression was decreased in OA Model rabbits, whereas
\end{abstract}

Correspondence to: Professor Xiaoling Fu, Department of Orthopedics, The Second Affiliated Hospital of Nanchang University, 1 Minde Road, Nanchang, Jiangxi 330006, P.R. China E-mail: fxiaoingd@163.com

Key words: osteoarthritis, $\gamma$-glutamyl carboxylase, matrix Gla protein, inflammation overexpression of GGCX was able to promote carboxylation of MGP, reduce inflammation, decrease MMP-13 expression and regulate collagen expression. The results also indicated that GGCX may serve as a therapeutic target for OA.

\section{Introduction}

Osteoarthritis (OA) is a chronic disabling disease that is induced by primary or secondary articular chondrocyte degeneration or structural disorder. It is the most common form of arthritis and affects millions of people worldwide, with the most common symptoms being joint pain and stiffness (1). OA occurs when the protective cartilage on the ends of bones wears down over time. Bone hyperplasia is a major pathological feature of OA, which leads to joint destruction and deformity (2). OA is most often diagnosed in middle-aged or elderly populations. As aging populations rise, the incidence rate of OA is now at an epidemic level worldwide, particularly in China $(3,4)$. Current treatments only provide symptomatic relief and include exercise, efforts to decrease joint stress, support groups and pain medications.

Although aging is considered to be one of the major pathological causes for the onset of OA (5), the exact underlying mechanisms that control OA development are yet to be elucidated. Matrix Gla protein (MGP) is a calcification inhibitor that must be carboxylated by vitamin $\mathrm{K}$ to function (6). $\gamma$-glutamyl carboxylase (GGCX) is a key enzyme that regulates the carboxylation of cartilage MGP, and carboxylated (c)MGP and uncarboxylated (un)MGP are detectable in cartilage cells. It has been suggested previously that circulating ucMGP may be elevated in patients with OA (7), and GGCX was apparently decreased in cartilage tissue from patients with OA $(8,9)$.

Inflammation has been identified as an important process in OA. Proinflammatory cytokines, including interleukin-1 $\beta$ (IL-1 $\beta)$ and tumor necrosis factor $\alpha(\mathrm{TNF}-\alpha)$, are critical markers for the diagnosis and treatment of OA (10). Collagen type $\mathrm{X}$ expression is typically upregulated in OA cartilage tissue, whereas collagen type II expression has been reported to be decreased in OA, which may be caused by the degeneration of cartilage cells (11). Matrix metalloproteinase (MMP)-13 is expressed in the skeleton and is required to restructure the collagen matrix for bone mineralization. In the present study, an OA model was produced by anterior cruciate ligament 
transection (ACLT) in rabbits. A GGCX-overexpression plasmid was locally injected into the joint fluid and the protection of GGCX overexpression against OA was investigated and inflammatory factors and collagen levels were detected. This study may provide experimental evidence for the clinical application of virus injection in the treatment of OA.

\section{Materials and methods}

Animal model and treatments. A total of 48 male Japanese white rabbits (age, 3 months; weight, $3 \pm 0.5 \mathrm{~kg}$ ) were obtained from the Animal Center of Nanchang University (Nanchang, China) and raised in a $12 \mathrm{~h}$ light/dark cycle at $22 \pm 3^{\circ} \mathrm{C}$ and a humidity of 40-60\%, with access to food and water ad libitum. All the experiments were approved by Ethics Committee of Nanchang University (Nanchang, China). The rabbits were divided into 4 groups (n=12/group): i) OA model + GGCX overexpression plasmid (GGCX); ii) OA model + saline (Model); iii) OA model + empty vector (Vector); and iv) Sham negative control (Sham). The OA model was produced by ACLT surgery, as previously described (12); briefly, following anesthesia by $3 \%$ pentobarbital sodium $(30 \mathrm{mg} / \mathrm{kg}$ ) ear vein injection, the right knee joint in each rabbit was exposed and the anterior cruciate ligament was cut. The joint cavity was blocked by suture and disinfected with iodophor. Sham control rabbits received surgery, but the anterior cruciate ligament was not cut. At 8 days post-surgery, the rabbits received lentivirus-carried GGCX overexpression plasmid, empty vector or saline treatments, locally injected into the joint cavity $(0.2 \mathrm{ml})$. Joint fluid was obtained using a syringe from each rabbit at weeks $2,4,6$ and 8 and stored at $-80^{\circ} \mathrm{C}$ until use. At week 8 following the injections, all animals were sacrificed by decapitation following anesthesia by $3 \%$ pentobarbital sodium (30 mg/kg). Joint fluid and articular tissues were collected for enzyme linked immunosorbent assay (ELISA), histological staining and RNA/protein extraction.

ELISA. ucMGP (cat. no. JL32168, J\&L Biological, Shanghai, China), cMGP (cat. no. JL32118, J\&L Biological), MMP-13 (cat. no. SEA099Rb, Cloud-Clone Corp., Wuhan, China), collagen type X (cat. no. MBS725868; MyBioSource, Inc., San Diego, CA, USA), collagen type II (cat. no. SEA572Rb, Cloud-Clone Corp.), TNF- $\alpha$ (cat. no. SEA133Rb, Cloud-Clone Corp.) and IL-1 $\beta$ (cat. no. E-EL-RB0013c, Elabscience, Houston, TX, USA) were detected in joint fluid (10 $\mu$ l) by ELISA method, according to the manufacturer's instructions.

Immunohistochemistry, safranin O-fast green staining and Giemsa staining. Articular tissues were fixed in $4 \%$ paraformaldehyde for $\sim 1$ week at $4^{\circ} \mathrm{C}$, cryoprotected in $30 \%$ sucrose for $1 \mathrm{~h}$ at $4^{\circ} \mathrm{C}$ and sectioned into $20 \mu \mathrm{m}$ thick sections with a frozen microtome. Immunostaining of histological sections was performed using monoclonal antibodies against GGCX (1:100; cat. no. ab107507; Abcam, Cambridge, UK), ucMGP (1:100; cat. no. ab108225; Abcam), anti-cMGP (1:100; cat. no. ab12416; Abcam), MMP-13 (1:100; cat. no. bs-10581R; BIOSS, Beijing, China), collagen type X (1:100; cat. no. bs-0554R; BIOSS), collagen type II (1:100; cat. no. bs-8859R; BIOSS), TNF- $\alpha$ (1:100; cat. no. ab179675; Abcam) and IL-1 $\beta$ (1:100; cat. no. ab200478;
Abcam). Endogenous peroxidase activity was blocked with $3 \%(\mathrm{v} / \mathrm{v}) \mathrm{H}_{2} \mathrm{O}_{2}$ for $5 \mathrm{~min}$ at room temperature. Subsequently, tissues were incubated with primary antibodies overnight at $4^{\circ} \mathrm{C}$, followed by incubation with horseradish peroxidase (HRP)-labeled goat anti-rabbit IgG secondary antibody (1:10,000; cat. no. A16104SAMPLE; Thermo Fisher Scientific, Inc., Waltham, MA, USA) for $30 \mathrm{~min}$ at room temperature and visualized with 3,3'-diaminobenzidine chromogen for $3 \mathrm{~min}$ at room temperature.

Safranin O-fast green staining was carried out to detect surface cartilage damage and Giemsa staining was performed to detect cartilage tissue. Tissues were fixed in $4 \%$ paraformaldehyde for $\sim 1$ week at $4{ }^{\circ} \mathrm{C}$, cryoprotected in $30 \%$ sucrose for $1 \mathrm{~h}$ at $4^{\circ} \mathrm{C}$ and sectioned into $20 \mu \mathrm{m}$ thick sections with a frozen microtome. For safranin O-fast green staining, slides were first stained with Weigert's iron hematoxylin working solution for $10 \mathrm{~min}$. Slides were subsequently washed in running tap water for $10 \mathrm{~min}$ and stained with fast green solution for $5 \mathrm{~min}$. Slides were then rinsed quickly with $1 \%$ acetic acid solution for no more than $10-15 \mathrm{sec}$ and stained with $0.1 \%$ safranin $\mathrm{O}$ solution for $5 \mathrm{~min}$. For Giemsa staining, fixed slides were stained with Giemsa stain for $5 \mathrm{~min}$. Both stains were performed at room temperature and images were taken under a light microscope in at least five fields of view with magnification, $\mathrm{x} 200$.

Reverse transcription-quantitative polymerase chain reaction $(R T-q P C R)$. Total RNA was extracted from articular tissues $(10 \mathrm{mg})$ using a TRIzol kit (Thermo Fisher Scientific, Inc.). RNA concentrations were determined spectrophotometrically, and $1 \mu \mathrm{g}$ total RNA was reverse transcribed into cDNA using an Avian Myeloblastosis Virus Reverse-Transcriptase kit (Promega Corporation, Madison, WI, USA). RT-qPCR was performed using the TB Green ${ }^{\mathrm{TM}}$ Fast qPCR Mix (Takara Biotechnology Co., Ltd., Dalian, China). PCR primer sequences were as follows: GAPDH forward, 5'-GTCTGCCACGATAACACC-3' and reverse, 5'-CAATAC AACAAGCCCACTC-3'; GGCX forward, 5'-CTTGTTGCG AAAGCTCTAT-3' and reverse, 5'-GATTTGACTCAGGAG GATTAG-3'; MMP-13 forward, 5'-CCCCAACCCTAAACA TCC-3' and reverse, 5'-AACAGCTCCGCATCAACC-3'; TNF- $\alpha$ forward, 5'-GCCGGATCGTGCAGTTCG-3' and reverse, 5'-TCCAAGGTAGCGGTCGTG-3'; IL-1 $\beta$ forward, 5'-GCTGAACCTTAGTACCCTTGT-3' and reversem, 5'-AGT TTCTGTGGCGTCTGG-3'; collagen type $\mathrm{X}$ forward, 5'-TCA AAGGGCACTATCAACT-3' and reverse, 5'-TGTTTGGTA TCGCTCAGTA-5'; collagen type II forward, 5'-CAACAA CCAGATCGAGAGCA-3' and reverse 5'-CGGTCTCCATGT TGCAGAA-3'. The amplification reactions were performed with an Applied Biosystems 7500 Real-Time PCR System (Applied Biosystems; Thermo Fisher Scientific, Inc., Waltham, MA, USA), with initial denaturation at $95^{\circ} \mathrm{C}$ for $10 \mathrm{~min}$, followed by 40 cycles of a two-step PCR at $95^{\circ} \mathrm{C}$ for $15 \mathrm{sec}$ and $60^{\circ} \mathrm{C}$ for $1 \mathrm{~min}$. The $2^{-\Delta \Delta C q}$ method was used to determine the amount of target, normalized to the endogenous reference, GAPDH, as previously described (13).

Western blotting. Protein was extracted from articular tissue for western blotting as previously described (14). Protein was isolated from $10 \mathrm{mg}$ articular tissue using a protein isolation 


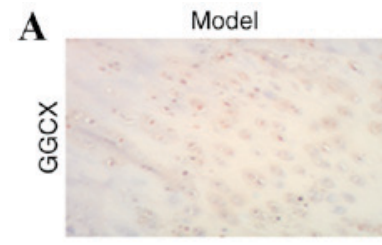

B

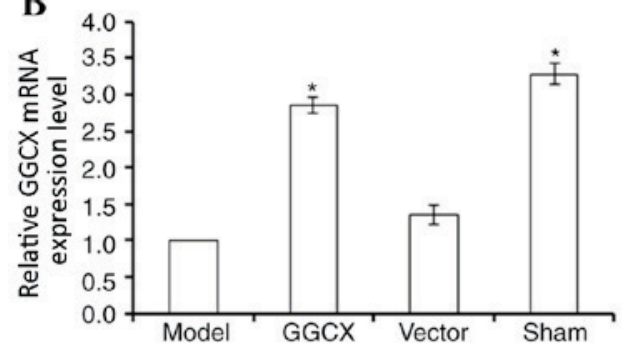

GGCX

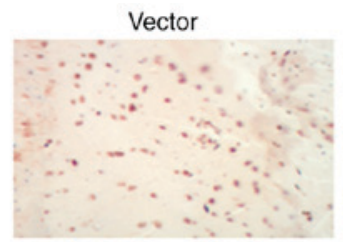

C
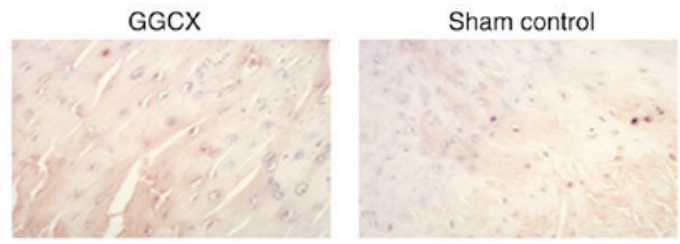

Sham contro

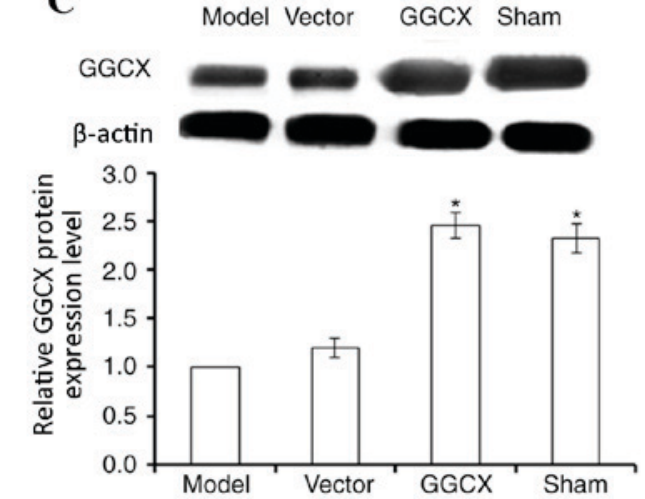

Figure 1. Injections with lentiviral-carried GGCX-overexpression plasmid promote GGCX expression in articular tissues in ATLC model rabbits. (A) Immunohistochemistry of articular tissue (magnification, x200); Tissues were probed with anti-GGCX antibody and counter stained with hematoxylin and eosin. (B) Relative expression of GGCX was determined by reverse transcription-quantitative polymerase chain reaction for GGCX. (C) Western blotting and densitometric analysis of GGCX protein expression in the four treatment groups; expression levels were normalized to $\beta$-actin. " $\mathrm{P}<0.05$ vs. Sham group; ${ }^{\#} \mathrm{P}<0.05$ vs. Model group. ATLC, anterior cruciate ligament transection; GGCX, $\gamma$-glutamyl carboxylase.

kit (ReadyPrep; GE Healthcare Life Sciences). Protein concentration was determined using a bicinchoninic assay kit (Thermo Fisher Scientific, Inc.). A total of $20 \mu \mathrm{g}$ protein was loaded into each lane and separated via SDS-PAGE on a $12 \%$ gel and transferred onto nitrocellulose membranes. Subsequently, membranes were blocked in 5\% skim milk for $2 \mathrm{~h}$ in room temperature and incubated with the following primary antibodies overnight at $4^{\circ} \mathrm{C}$ : Anti-GGCX (1:200; cat. no. ab107507; Abcam), anti-ucMGP (1:200; cat. no. ab108225; Abcam), anti-cMGP (1:200; cat. no. ab12416, Abcam) and anti- $\beta$-actin (1:1,000; cat. no. 4970; Cell Signaling Technology, Inc., Danvers, USA); membranes were incubated with primary antibodies overnight at $4^{\circ} \mathrm{C}$. The nitrocellulose membranes were washed three times and incubated with HRP-labeled goat anti-rabbit IgG secondary antibody (1:10,000, cat. no. A16104SAMPLE; Thermo Fisher Scientific, Inc.) at $4^{\circ} \mathrm{C}$ for $2 \mathrm{~h}$. Protein bands were visualized using an enhanced chemiluminescence kit (Thermo Fisher Scientific, Inc.) and the blots were scanned using a ChemiDoc XRS (Bio-Rad Laboratories, Inc., Hercules, CA, USA). Protein expression was normalized to $\beta$-actin and densitometric analysis was performed by Image J Software version 7.0 (National Institutes of Health, Bethesda, MD, USA).

Statistical analysis. Data are presented as the mean \pm standard deviation. One-way analysis of variance with Bonferroni post-hoc test for multiple comparisons was performed. $\mathrm{P}<0.05$ was considered to indicate a statistically significant difference.

\section{Results}

GGCX overexpression promotes GGCX expression in $O A$ model rabbits. The potential roles of GGCX in OA were examined in an ACLT-induced OA rabbit model by locally injecting lentivirus carrying GGCX overexpression vector into the joint fluid. GGCX mRNA expression was detected by RT-qPCR, and protein expression was detected by immunohistochemistry (Fig. 1A) and western blot analysis. GGCX protein and mRNA expression was significantly decreased in the OA model group compared with expression levels in Sham control rabbit articular cartilage (Fig. 1B and C). No significant difference was identified for GGCX mRNA or protein expression in OA Model rabbits treated with the empty vector compared with untreated Model rabbits, whereas those receiving injections of the GGCX-overexpression vector exhibited significantly increased GGCX expression at both the mRNA and protein levels in articular cartilage compared with the Model group. These data suggested that local injection of GGCX overexpression plasmid was a useful approach for promoting GGCX expression.

Morphological changes. Articular cartilage from rabbits in each of the four groups was stained with safranin $\mathrm{O}$-fast green. The articular cartilage appeared normal in the Sham control group, which was characterized by a smooth surface and with evenly organized cartilage cells in clear layers; aggregated cells were not observed and tide line was complete with matrix evenly stained (Fig. 2). By contrast, morphological changes were clearly observed in the OA Model and the empty vector groups, in which severe cartilage injury and large-scale cartilage fibrosis was observed, and matrix was damaged in most of the layers. GGCX overexpression appeared to reduce the morphological changes caused by ACLT (Fig. 2). Cartilage cells in tibia were normally distributed in Sham control group (Fig. 3); the cells were evenly and orderly arranged with clear layers, a complete tide line and normal staining of matrix. In the OA 


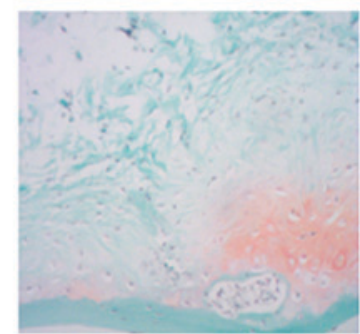

Model

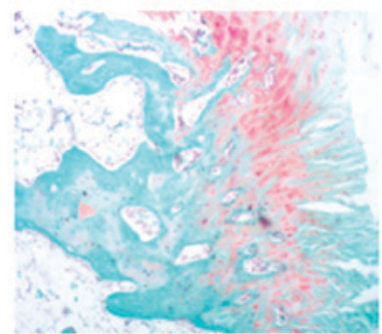

Vector

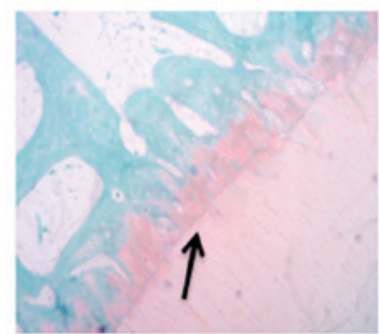

GGCX

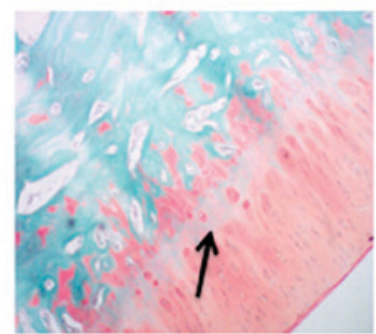

Sham control

Figure 2. GGCX overexpression reverses anterior cruciate ligament transection-induced morphological changes of articular cartilage, as detected by Safranin O-fast green staining (magnification, x200). The articular cartilage appeared normal in the Sham control group and GGCX overexpression group, which was characterized by a smooth surface and with evenly organized cartilage cells in clear layers; aggregated cells were not observed and tide line was complete with matrix evenly stained, as indicated by the arrows. By contrast, changes in morphology were observed in the Model and Vector groups, indicating cartilage injury and fibrosis. GGCX, $\gamma$-glutamyl carboxylase.

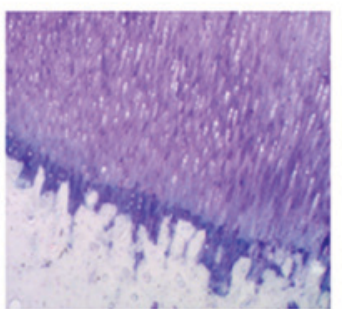

Model

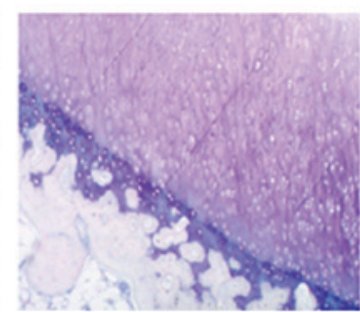

Vector

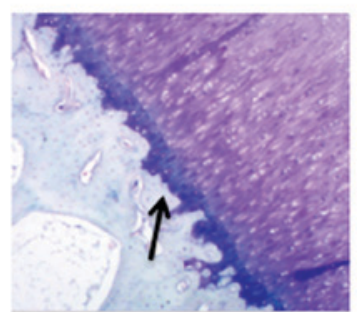

GGCX

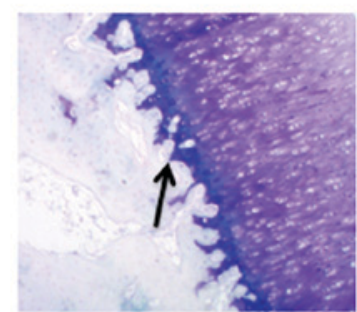

Sham control

Figure 3. GGCX overexpression reverses anterior cruciate ligament transection-induced morphological changes of tibial cartilage, as detected by Giemsa staining (magnification, x200). Cartilage cells were normally distributed in the Sham and GGCX overexpression group. Orderly arranged cells with clear layers, a complete tide line (as indicated by arrows) and normal staining of matrix were observed. In the OA Model group and empty vector group, although the tide line was complete, most layers of the matrix exhibited mild injury. GGCX, $\gamma$-glutamyl carboxylase.

Model group and empty vector group, although the tide line was complete, most layers of the matrix exhibited mild injury. However, GGCX overexpression appeared to attenuate the morphological changes caused by ATLC.

GGCX overexpression decreases ucMGP and increases cMGP expression levels in OA model rabbits. Injection of the GGCX overexpression plasmid was demonstrated to increase GGCX expression in articular cartilage and joint fluid, as determined by ELISA. Thus, it was investigated whether MGP levels were also affected by GGCX overexpression. In OA Model rabbits and in rabbits treated with the empty vector, ucMGP expression levels gradually increased between week 2 and week 8, compared with the control (Fig. 4A). Rabbits overexpressing GGCX exhibited a significant decrease in ucMGP expression level at week 8, compared with rabbits in the Model group. By contrast, OA Model rabbits exhibited gradually decreasing cMGP expression levels between weeks 2 and 8 (Fig. 4B). GGCX overexpression vector injection promoted the recovery of cMGP to normal level (week 8), whereas the empty vector did not influence cMGP, compared with Model group.

Immunohistochemistry and western blot analysis confirmed the results obtained from ELISA. OA model rabbits exhibited an increase in ucMGP expression (Fig. 4C and D) and a decrease in cMGP expression levels at week 8 post-surgery (Fig. 4C and E). By contrast, GGCX overexpression reversed the abnormalities caused by the ATLC model.
GGCX overexpression reduces inflammation induced by $A T L C$. Representative images of the joints in each group are presented in Fig. 5. The articular cartilage of the sham group was observed to be intact without injury. By contrast, the joint fluid in the model group was cloudy and the articular cartilage was damaged with evidence of inflammation. The vector and model group morphologies were indistinguishable. In the GGCX group, the injured joints appeared to have recovered to a normal level. TNF- $\alpha$ (Fig. 6A) and IL-1 $\beta$ (Fig. 2B) expression levels were detected by ELISA, immunohistochemistry (Fig. 6C) and RT-qPCR (Fig. 6D). TNF- $\alpha$ concentration increased between week 2 and week 8 post-surgery in the OA Model and empty vector groups (Fig. 6A). At week 8 , TNF- $\alpha$ mRNA and protein expression levels were increased in the OA Model group (Fig. 6C). By contrast, GGCX overexpression, but not empty vector, caused a decrease in ATLC-induced TNF- $\alpha$ expression (Fig. 6A, C and D). A similar trend was identified for IL-1 $\beta$ expression levels; OA Model rabbits exhibited increased IL-1 $\beta$ synovial fluid concentration levels, protein expression and mRNA expression levels (Fig. 6B, C and E, respectively).

GGCX overexpression decreases collagen type $X$ and MMP-13 expression, but increases collagen type II expression in ATLC model rabbits. In the present study, OA Model rabbits exhibited a decrease in collagen type II synovial fluid concentration levels, but an increase in collagen type $\mathrm{X}$ concentration levels between week 2 and 

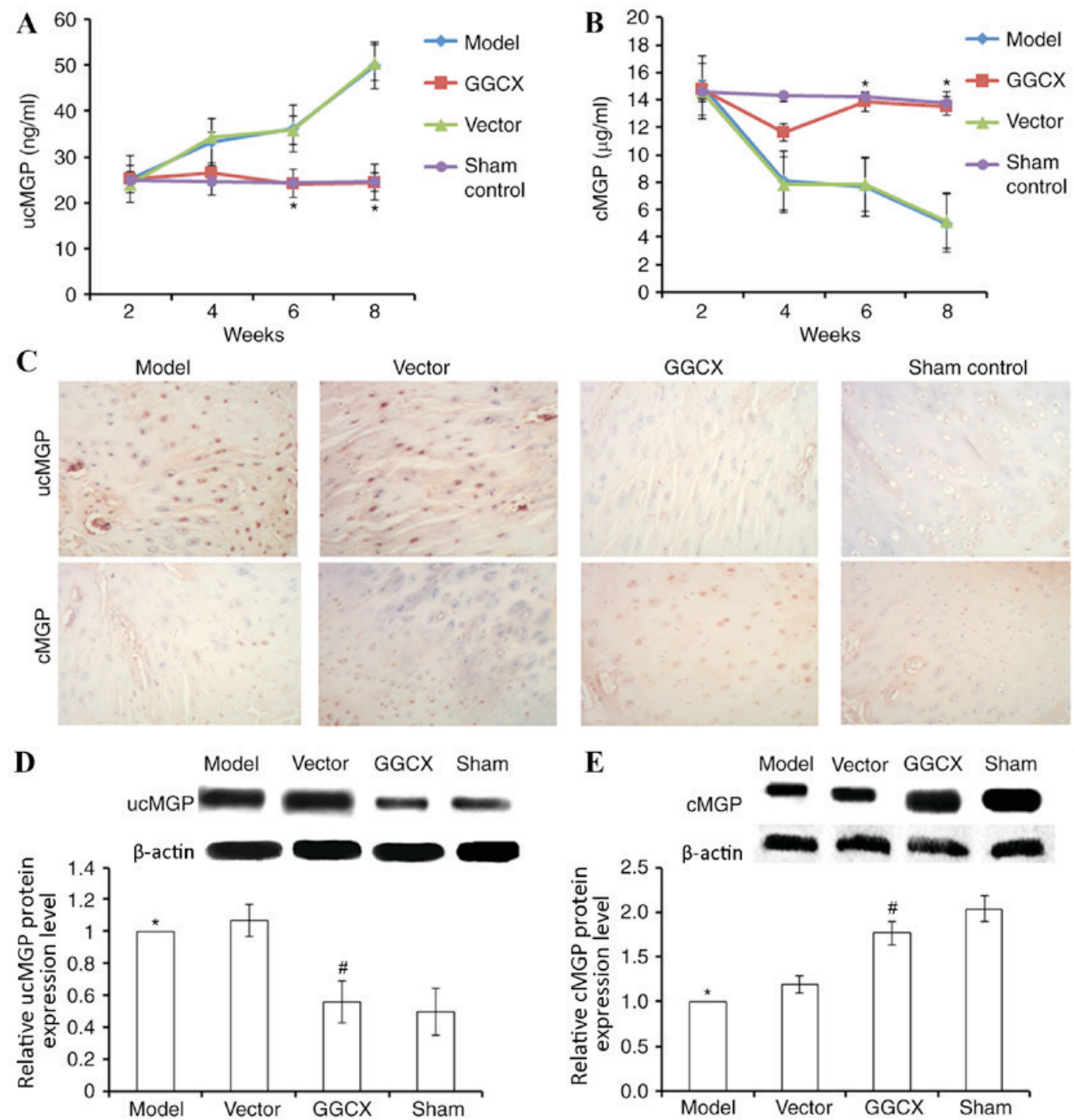

Figure 4. GGCX overexpression reduces ucMGP and increased cMGP expression in ATLC Model rabbits. (A) ELISA detection of ucMGP in joint fluid. (B) ELISA detecting cMGP in joint fluid ${ }^{*} \mathrm{P}<0.05$ vs. Model. (C) Immunohistochemistry of articular tissues. Magnification, $\mathrm{x} 200$. Detection of (D) ucMGP and (E) cMGP of articular tissues, as detected by western blot analysis. "P $<0.05$ vs. Sham group; ${ }^{\prime} \mathrm{P}<0.05$ vs. Model group. ATLC, anterior cruciate ligament transection; c, carboxylated; GGCX, $\gamma$-glutamyl carboxylase; MGP, matrix Gla protein; uc, uncarboxylated.

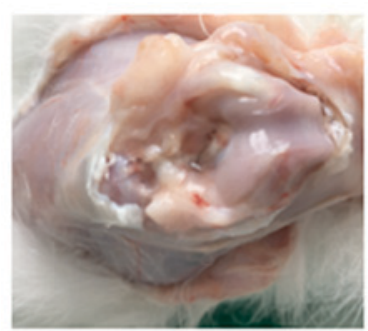

Model

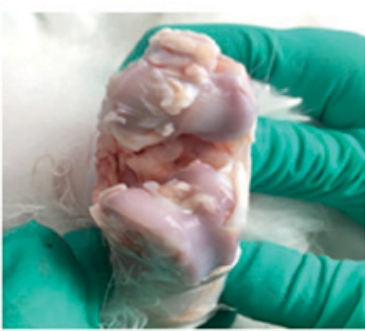

Vector

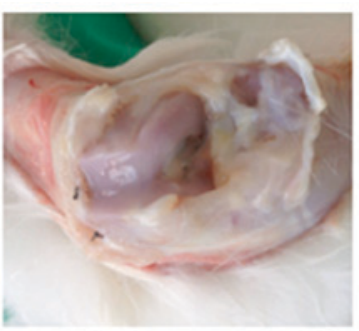

GGCX

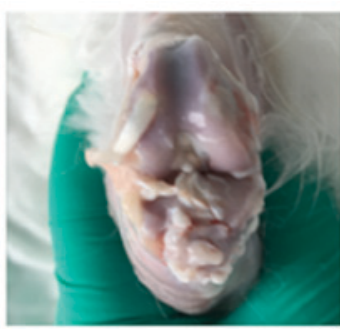

Sham control

Figure 5. Representative joint images of each experimental group. ATLC, anterior cruciate ligament transection; GGCX, $\gamma$-glutamyl carboxylase.

week 8 post-surgery (Fig. 7A and B, respectively). At week 8 post-surgery, OA Model rabbits also exhibited a decrease in collagen type II and an increase in collagen type $\mathrm{X}$ protein and mRNA expression levels (Fig. 7C-E). By contrast, GGCX overexpression reversed the abnormalities caused by ATLC-induced OA.
The levels of MMP-13 expression were also examined using similar methods. OA Model rabbits exhibited gradually increasing MMP-13 concentration levels in the synovial fluid between weeks 2 and 8 post-surgery compared with the Sham group (Fig. 8A). At week 8, OA Model rabbits also exhibited increased MMP-13 mRNA and protein expression levels 

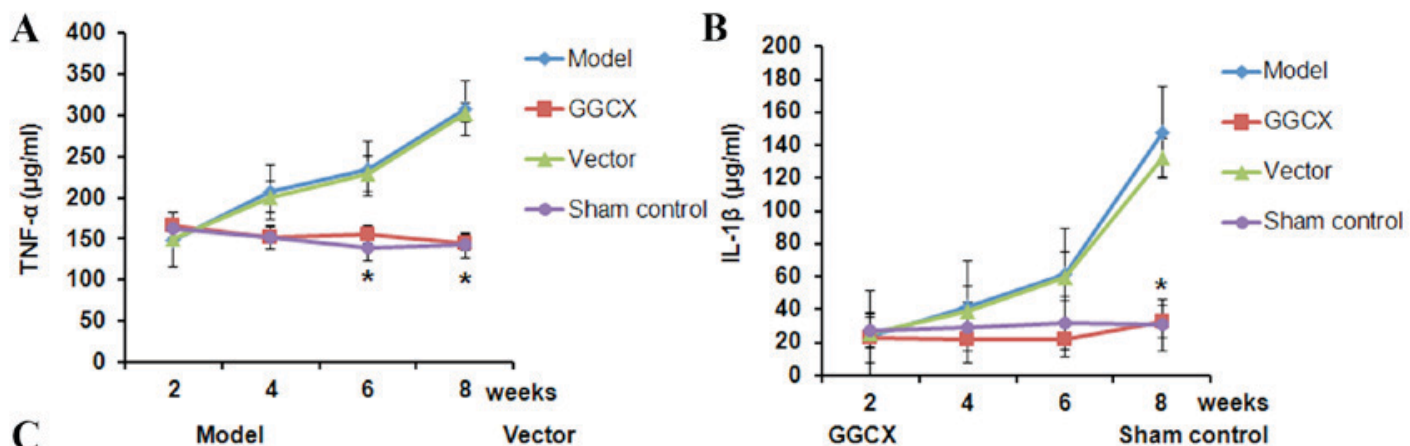

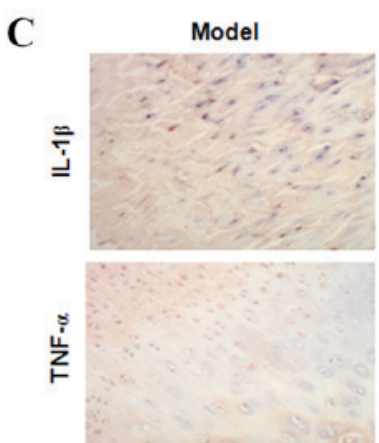

D
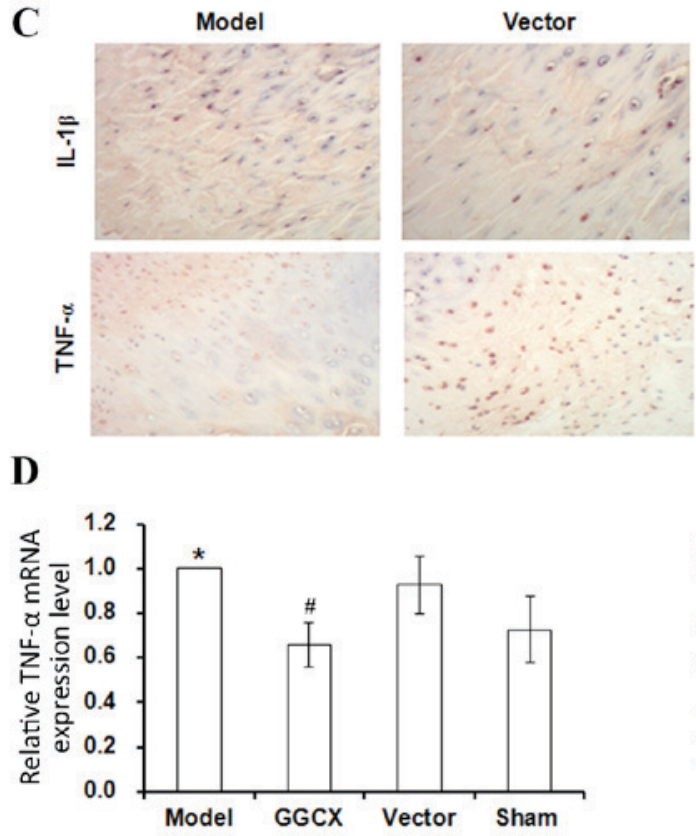

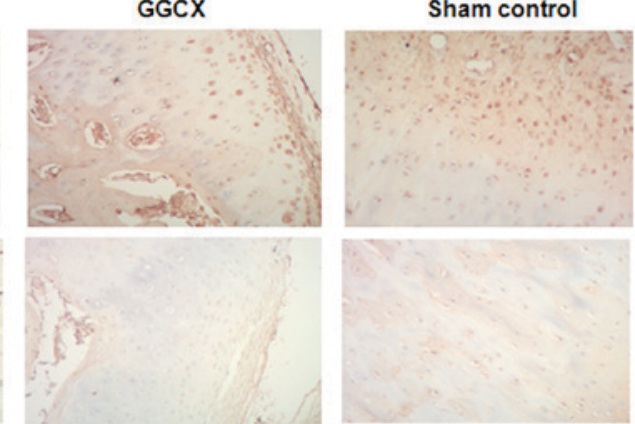

E

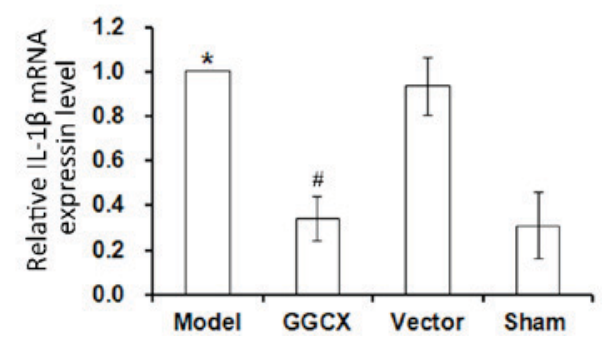

Figure 6. GGCX overexpression reduces IL- $1 \beta$ and TNF- $\alpha$ expression in ATLC model rabbits. (A) ELISA detection of TNF- $\alpha$ in joint fluid. (B) ELISA detection of IL-1 $\beta$ in joint fluid. ${ }^{*} \mathrm{P}<0.05$ vs. Model. (C) Immunohistochemistry of articular tissues. Magnification, $\mathrm{x} 200$. Detection of (D) TNF- $\alpha$ and (E) IL-1 $\beta$ mRNA expression by reverse transcription-quantitative polymerase chain reaction. ${ }^{*} \mathrm{P}<0.05$ vs. Sham group; ${ }^{~} \mathrm{P}<0.05$ vs. Model group. ATLC, anterior cruciate ligament transection; GGCX, $\gamma$-glutamyl carboxylase; IL, interleukin; TNF, tumor necrosis factor.

compared with the Sham group (Fig. 8B and C, respectively). By contrast, GGCX overexpression reversed the abnormalities caused by ATLC surgery.

\section{Discussion}

Primary OA is characterized by restricted articular activity, pain and hyperosteogeny (15). Certain treatments, including non-steroidal analgesic and anti-inflammatory drugs, and amino glucose capsules, were reported to alleviate the symptoms, but with only temporal effects $(16,17)$. Therefore, effective treatment for OA is still urgently required. In the present study, the overexpression of GGCX was demonstrated to mitigate ATLC-induced damage of articular cartilage. The potential mechanisms may be related to the reduction of inflammation, as well as an increase in cMGP and a decrease in ucMGP concentration in joint fluid. GGCX is a key enzyme responsible for the carboxylation of cartilage MGP (18). In normal articular cartilage, cMGP binds to calcium and fetuin to form an MGP-fetuin-calcium complex. This complex inhibits cartilage calcification and the formation of calcium crystals, which was reported to elicit the degeneration of cartilage cells (19). The present study demonstrated that GGCX overexpression promoted the carboxylation of MGP in OA model rabbits.
Inflammation has been reported be an important process in OA (20). IL-1 $\beta$ and TNF- $\alpha$ are the major inflammatory cytokines in OA development. As previously reported, IL-1 $\beta$ and TNF- $\alpha$ levels were apparently elevated in OA $(21,22)$. In the present study, mRNA and protein levels of IL-1 $\beta$ and TNF- $\alpha$ were increased in OA Model rabbit joint synovial fluid. GGCX overexpression was revealed to reduce the expression of these inflammatory factors and joint inflammation in the ATLC Model rabbits.

In a previous study, collagen type X expression was revealed to be increased in OA cartilage tissue (23). In the present study, three approaches consistently demonstrated that GGCX overexpression in OA Model rabbits led to increased collagen type II and decreased collagen type X expression levels. MMPs are capable of degrading all kinds of extracellular matrix proteins, including collagen type II, and increased MMP expression may have detrimental effects on cartilage cells (24). The present study used three methods to demonstrate that MMP-13 synthesis was increased in OA Model rabbits, whereas it was reduced by GGCX overexpression.

The present study also detected IL- $1 \beta$, TNF- $\alpha$, Collagen type X, Collagen type II and MGPat different time points by ELISA. The expression of these proteins was not clearly affected in the second week post-surgery; however, the differences were 

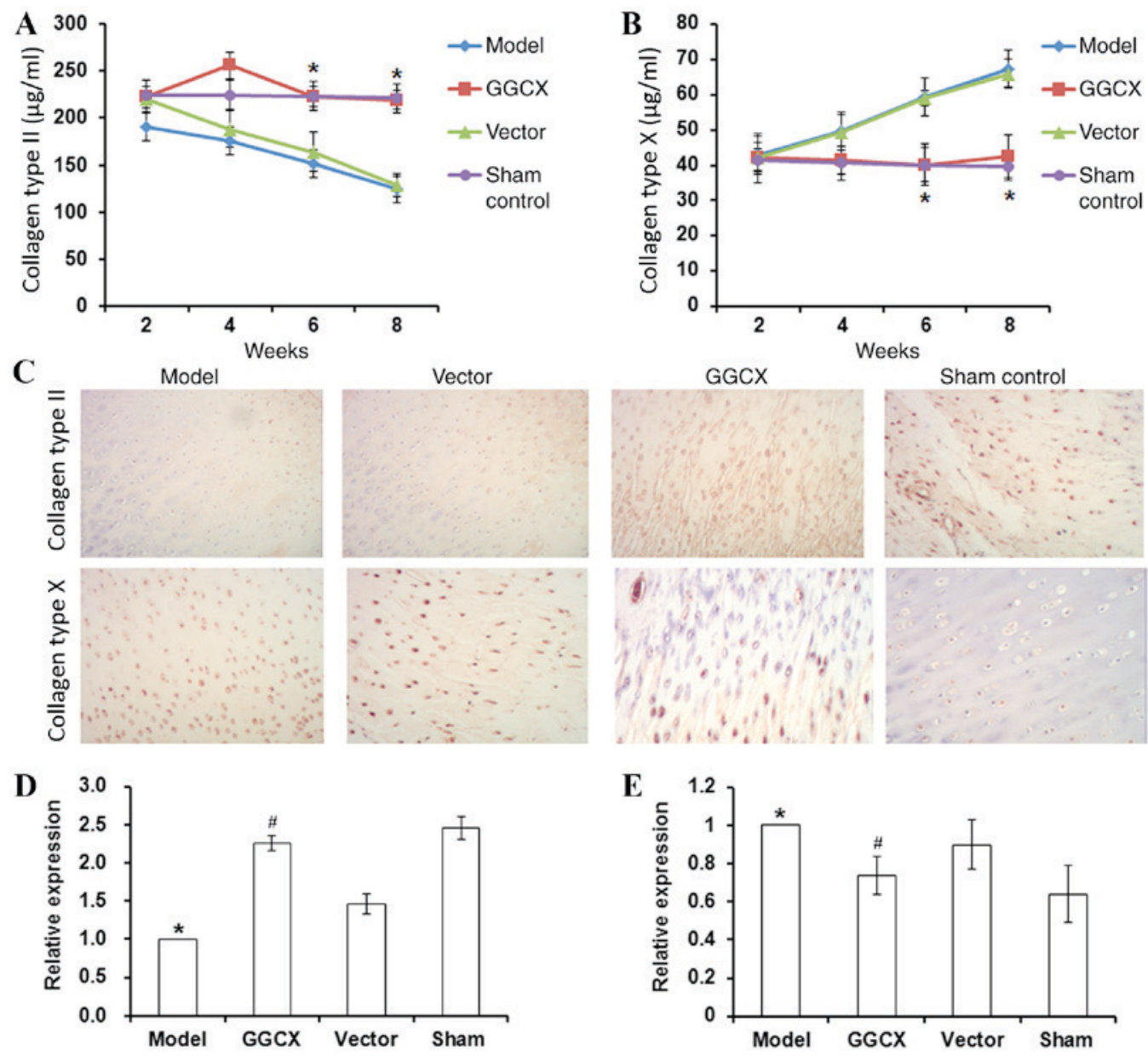

Figure 7. GGCX overexpression reverses abnormalities in collagen type II and collagen type X expression in ATLC Model rabbits. (A) ELISA detection of collagen type II. (B) ELISA detection of collagen type $\mathrm{X} .{ }^{*} \mathrm{P}<0.05$ vs. Model. (C) Immunohistochemistry. Magnification, x200. Detection of (D) collagen type II and (E) collagen type $\mathrm{X}$ mRNA expression by reverse transcription-quantitative polymerase chain reaction. ${ }^{*} \mathrm{P}<0.05$ vs. Sham group; ${ }^{\#} \mathrm{P}<0.05$ vs. Model group. ATLC, anterior cruciate ligament transection; GGCX, $\gamma$-glutamyl carboxylase.
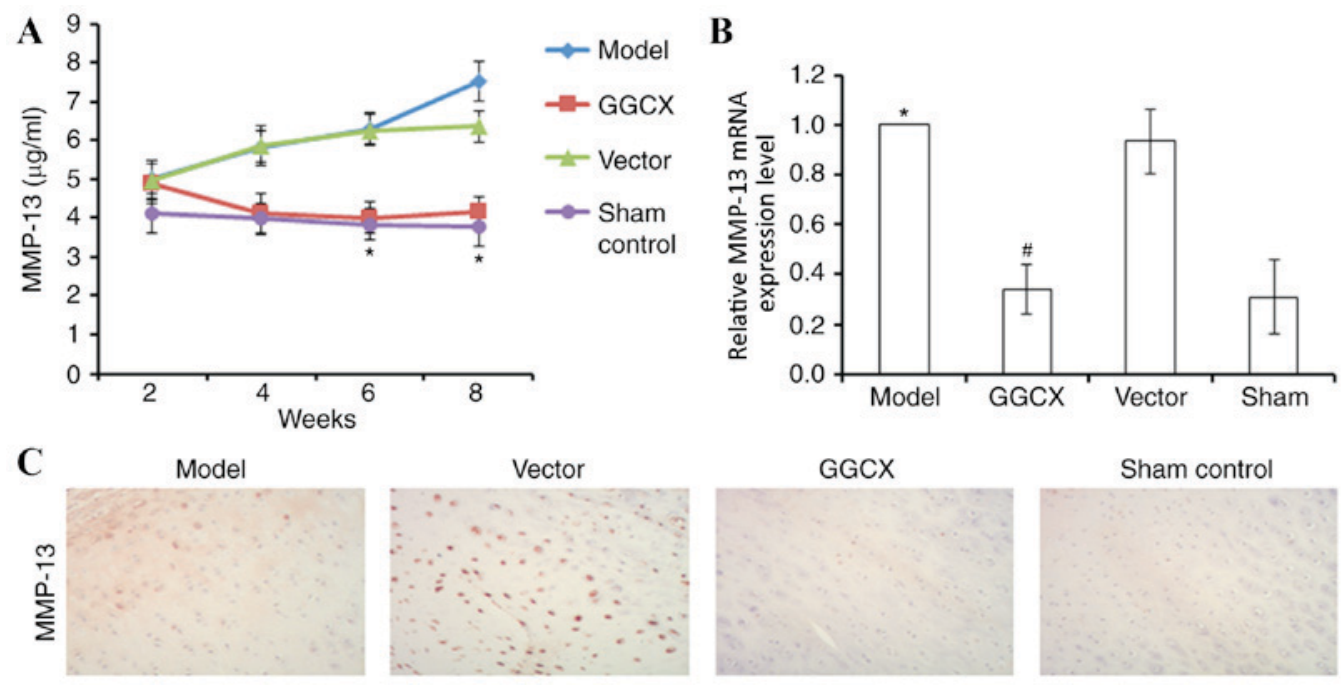

Figure 8. GGCX overexpression reduced ATLC-induced MMP-13 expression in Model rabbits. (A) ELISA. "P<0.05 vs. Model. (B) Reverse transcription-quantitative polymerase chain reaction. (C) Immunohistochemistry; magnification, $\mathrm{x} 200 .{ }^{*} \mathrm{P}<0.05$ vs. Sham group; ${ }^{~} \mathrm{P}<0.05$ vs. Model group. ATLC, anterior cruciate ligament transection; GGCX, $\gamma$-glutamyl carboxylase.

apparent at weeks 6 and 8 . In addition, the protective effects of GGCX overexpression against ATLC-induced articular injury were also demonstrated. A number of previous studies also indicated that growth arrest-specific 6 (Gas6) functioned in promoting the proliferation of cartilage cells $(25,26)$. GGCX promotes the carboxylation of MGP, and cMGP may exert its 
function through Gas6 to inhibit cartilage cell apoptosis (27). This data is consistent with the findings of the present study and indicates that GGCX overexpression has the potential to ameliorate OA injury. Morphological analysis by safranin $\mathrm{O}$-fast green and Giemsa staining revealed alterations in articular cartilage and tibia. Results from the present study indicated that GGCX overexpression may ameliorate the morphological changes induced by ATLC.

In conclusion, the present study investigated the effects of GGCX overexpression on ATLC-induced articular impairments. GGCX expression was demonstrated to be decreased in the OA Model, whereas subsequent overexpression of GGCX in OA Model rabbits was able to reduce inflammation, reduce collagen type $\mathrm{X}$ and MMP-13 expression, promote MGP carboxylation and increase collagen type II expression. Results from the present study indicate that GGCX may serve as an effective target for OA treatment.

\section{Acknowledgements}

This research was supported by The National Natural Science Foundation of China (grant no. 81301561).

\section{References}

1. Loeser RF, Goldring SR, Scanzello CR and Goldring MB: Osteoarthritis: A disease of the joint as an organ. Arthritis Rheum 64: 1697-1707, 2012.

2. Crema MD, Roemer FW, Felson DT, Englund M, Wang K, Jarraya M, Nevitt MC, Marra MD, Torner JC, Lewis CE and Guermazi A: Factors associated with meniscal extrusion in knees with or at risk for osteoarthritis: The multicenter osteoarthritis study. Radiology 264: 494-503, 2012.

3. Michael JW, Schlüter-Brust KU and Eysel P: The epidemiology, etiology, diagnosis, and treatment of osteoarthritis of the knee. Dtsch Arztebl Int 107: 152-162, 2010.

4. Zhang Y and Jordan JM: Epidemiology of osteoarthritis. Clin Geriatr Med 26: 355-369, 2010.

5. Geusens PP and van den Bergh JP: Osteoporosis and osteoarthritis: Shared mechanisms and epidemiology. Curr Opin Rheumatol 28: 97-103, 2016.

6. Shea MK, O'Donnell CJ, Vermeer C, Magdeleyns EJ, Crosier MD, Gundberg CM, Ordovas JM, Kritchevsky SB and Booth SL: Circulating uncarboxylated matrix gla protein is associated with vitamin $\mathrm{K}$ nutritional status, but not coronary artery calcium, in older adults. J Nutr 141: 1529-1534, 2011.

7. Silaghi CN, Fodor D, Cristea V and Craciun AM: Synovial and serum levels of uncarboxylated matrix Gla-protein (ucMGP) in patients with arthritis. Clin Chem Lab Med 50: $125-128,2012$.

8. Xi XF, Li XZ, Liu F, Fu NN, Ren Y, Yang XG and Zhang Y: Effects of short thrust needing plus Electroacupuncture intervention on cartilage tissue in rabbits with knee osteoarthritis. Zhen Ci Yan Jiu 41: 124-130, 2016 (In Chinese).

9. Shi Y, Chen W and Yan S: Study on effect of GGCX in knee osteoarthritis pathogenesis. Int J Clin Exp Med 9: 13657-13663, 2016.
10. Sokolove J and Lepus CM: Role of inflammation in the pathogenesis of osteoarthritis: Latest findings and interpretations. Ther Adv Musculoskelet Dis 5: 77-94, 2013.

11. Bello AE and Oesser S: Collagen hydrolysate for the treatment of osteoarthritis and other joint disorders: A review of the literature. Curr Med Res Opin 22: 2221-2232, 2006.

12. Boulocher C, Duclos ME, Arnault F, Roualdes O, Fau D, Hartmann DJ, Roger T, Vignon E and Viguier E: Knee joint ultrasonography of the ACLT rabbit experimental model of osteoarthritis: Relevance and effectiveness in detecting meniscal lesions. Osteoarthritis Cartilage 16: 470-479, 2008.

13. Livak KJ and Schmittgen TD: Analysis of relative gene expression data using real-time quantitative PCR and the 2(-Delta Delta C(T)) method. Methods 25: 402-408, 2001.

14. Kurz B, Domm C, Jin M, Sellckau R and Schünke M: Tissue engineering of articular cartilage under the influence of collagen I/III membranes and low oxygen tension. Tissue Eng 10: 1277-1286, 2004.

15. Arden N and Nevitt MC: Osteoarthritis: Epidemiology. Best Pract Res Clin Rheumatol 20: 3-25, 2006.

16. Phillips S, Silvia Li C, Phillips M, Bischoff M, Ali P, Chahal J, Snider M and Bhandari M: Treatment of osteoarthritis of the Knee with bracing: A scoping review. Orthop Rev (Pavia) 8: $6256,2016$.

17. Anandacoomarasamy A and March L: Current evidence for osteoarthritis treatments. Ther Adv Musculoskelet Dis 2: 17-28, 2010.

18. Viegas CS, Cavaco S, Neves PL, Ferreira A, João A, Williamson MK, Price PA, Cancela ML and Simes DC: Gla-rich protein is a novel vitamin $\mathrm{K}$-dependent protein present in serum that accumulates at sites of pathological calcifications. Am J Pathol 175: 2288-2298, 2009.

19. Lorenzen JM, Martino F, Scheffner I, Bröcker V, Leitolf H, Haller $\mathrm{H}$ and Gwinner W: Fetuin, matrix-Gla protein and osteopontin in calcification of renal allografts. PLoS One 7: e52039, 2012.

20. Berenbaum F, Griffin TM and Liu-Bryan R: Metabolic regulation of inflammation in osteoarthritis. Arthritis Rheumatol 69: 9-21, 2017.

21. Martin MU and Wesche H: Summary and comparison of the signaling mechanisms of the Toll/interleukin-1 receptor family. Biochim Biophys Acta 1592: 265-280, 2002.

22. Chambers MG, Kuffner T, Cowan SK, Cheah KS and Mason RM: Expression of collagen and aggrecan genes in normal and osteoarthritic murine knee joints. Osteoarthritis Cartilage 10: 51-61, 2002.

23. Eerola I, Salminen H, Lammi P, Lammi M, von der Mark K, Vuorio E and Säämänen AM: Type X collagen, a natural component of mouse articular cartilage: Association with growth, aging, and osteoarthritis. Arthritis Rheum 41: 1287-1295, 1998.

24. Lu P, Takai K, Weaver VM and Werb Z: Extracellular matrix degradation and remodeling in development and disease. Cold Spring Harb Perspect Biol 3: pii: a005058, 2011.

25. Loeser RF, Varnum BC, Carlson CS, Goldring MB, Liu ET, Sadiev S, Kute TE and Wallin R: Human chondrocyte expression of growth-arrest-specific gene 6 and the tyrosine kinase receptor axl: Potential role in autocrine signaling in cartilage. Arthritis Rheum 40: 1455-1465, 1997.

26. Melaragno MG, Cavet ME, Yan C, Tai LK, Jin ZG, Haendeler J and Berk BC: Gas6 inhibits apoptosis in vascular smooth muscle: Role of Axl kinase and Akt. J Mol Cell Cardiol 37: 881-887, 2004.

27. Stenhoff J, Dahlbäck B and Hafizi S: Vitamin K-dependent Gas6 activates ERK kinase and stimulates growth of cardiac fibroblasts. Biochem Biophys Res Commun 319: 871-878, 2004. 